\title{
Inhalt / Contents 09/04
}

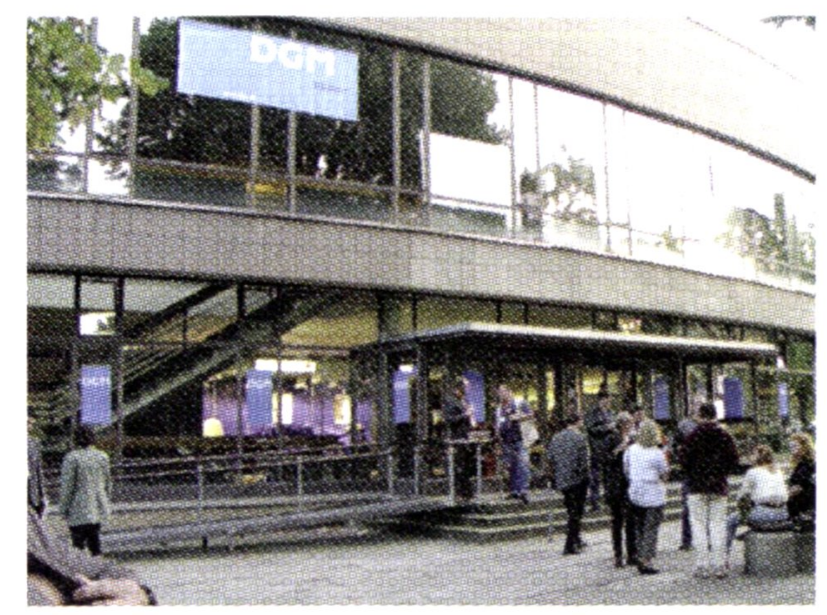

\section{P.D. Portella}

Bildbericht: Metallographie-Tagung

Berlin, 17.-19. September 2003

Photographical Report: Conference on Metallography

Berlin, September 17-19, 2003

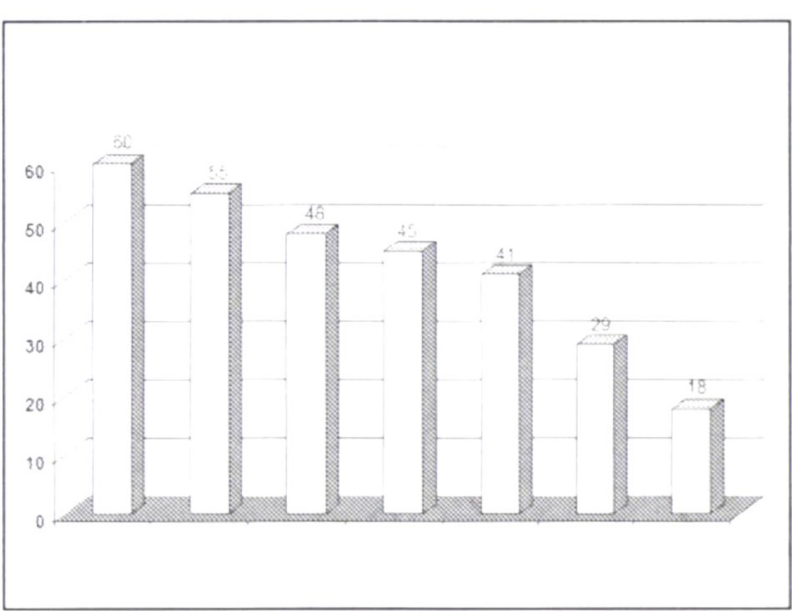

\section{W.-U. Kopp}

Metallographische Probenpräparation in

Deutschland 2003: Ergebnis einer Umfrage

Metallographic Sample Preparation in

Germany in 2003: The Result of an Inquiry

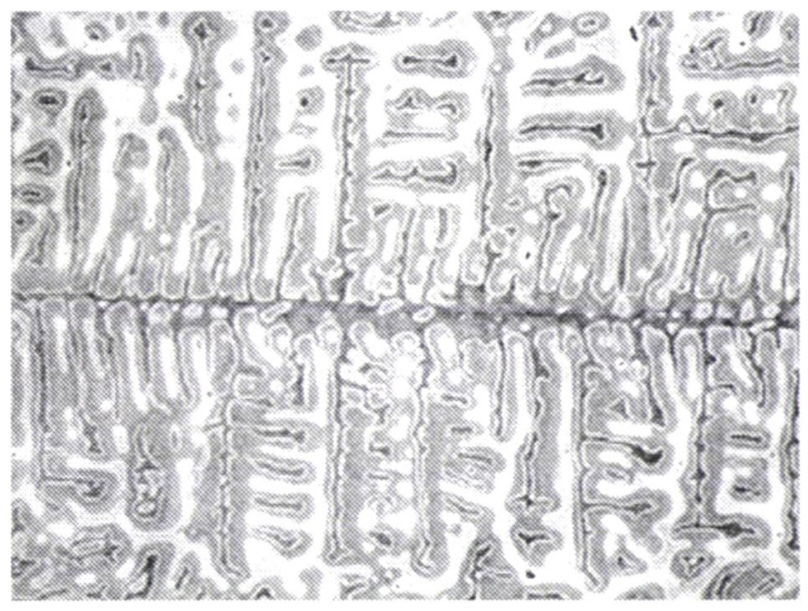

\section{E. Exner, M. Rettenmayr, C. Müller}

Complex Interface Geometries in

Phase Transformations

Komplexe Grenzflächengeometrien

bei Phasenumwandlungen

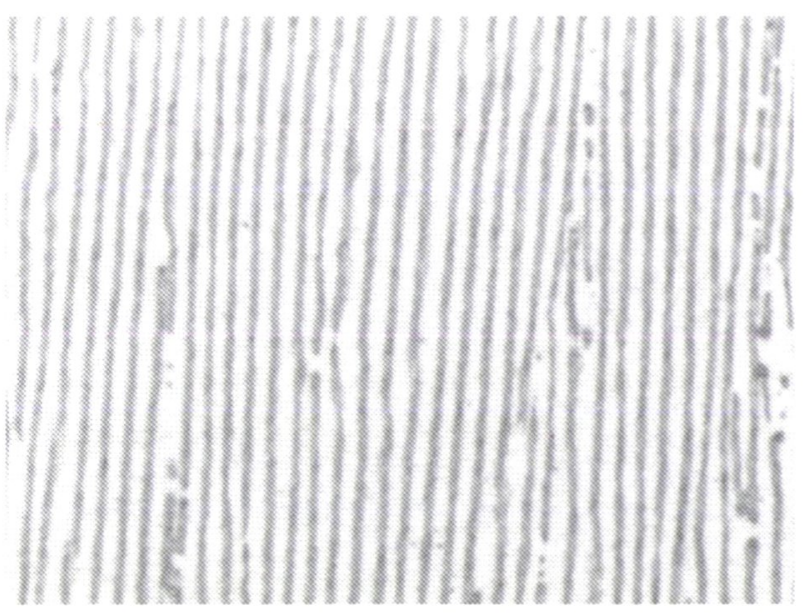

\section{Bruncko, I. Anzel, A. Križman}

Characterization of Directionally Solidified

Microstructure of Eutectic Pb-Sn Alloy

Charakterisierung eines gerichtet erstarrten

Gefüges einer eutektischen $\mathrm{Pb}$-Sn-Legierung

Literatur-Notizen/Literature Reviews

433,472

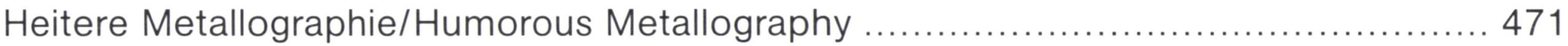

Diskussionsforum/Topic of Discussion

Veranstaltungskalender/Meeting Diary

Vorschau auf das nächste Heft unter:

Preview on next issue at:

http://www.materialography.de 


\section{Praktische Metallographie \\ Practical Metallography}

Gründer/Founders

Günter Petzow

Gerhard Reinacher +

Charlotte Wachau $\dagger$

\section{Herausgeber/Editor}

Prof. Dr. G. Petzow

\section{Schriftleiter}

\section{Editor in charge}

Prof. Dr.-Ing. F. Mücklich

Universität des Saarlandes

Lehrstuhl für Funktionswerkstoffe

Postfach 151150

D-66041 Saarbrücken

Telefon: +49/681 302-2048

Telefax: +49/681 302-4876

E-Mail: pm-editor@matsci.uni-sb.de

\section{Redaktion / Editor}

Dipl.-Ing. S. Mücklich

Zöblitzer Str. 10

D-09125 Chemnitz

Telefon: $+49 / 371 / 531-5384$

Telefax: +49/371/531-6179

E-Mail: silke.muecklich@mb.tu-

chemnitz.de

\section{HANSER}

Carl Hanser Verlag, München

\section{Wissenschaftlicher Beirat/Editorial Board}

Dr. C. Bagnall, MCS Associates Inc., Greensburg, PA (USA)

Dr. E. Bischoff, MPI für Metallforschung, Stuttgart

C. Bochert, Buehler GmbH, Düsseldorf

Prof. Dr. H.-E. Bühler, RWTH Aachen

V. Dietl, Lette Verein Berlin

Dr. G. Elssner, MPI für Metallforschung, Stuttgart

Prof. Dr. H.E. Exner, TU Darmstadt

Prof. Dr. M. Göken, Friedrich-Alexander-Univ.,

$$
\text { Erlangen-Nürnberg }
$$

Prof. Dr. E. Hornbogen, Ruhr-Universität Bochum

Prof. Dr. F. Jeglitsch, Montanuniversität Leoben

Prof. Dr. C.-K. Kim, KUT, Korea

Dr. H.-J. Klaar, RWTH Aachen

Prof. Dr. A. KneissI, Montanuniversität Leoben

Dr. W.-U. Kopp, Daisendorf

Prof. Dr. G. Liu, Univ. Beijing, P.R. China

Dr. J. Paul, Leica Vertrieb GmbH, Bensheim

Prof. Dr. M. Pohl, Ruhr-Universität Bochum

Prof. Dr. P.D. Portella, BAM, Berlin

Prof. Dr. Schmitt-Thomas, IST, München

Prof. Dr. G. Schneider, Bosch, Stuttgart

U. Täffner, MPI für Metallforschung, Stuttgart

Dr. J. Trempler, Martin-Luther-Univ. Halle-Wittenberg

Prof. Dr. H.-H. Uchida, Tokai Univ., Hiratsuka, Kanagawa

G. Vander Voort, Buehler Ltd., Illinois

Prof. Dr. B. Wielage, TU Chemnitz

Dr. H.-J. Wieland, Verein Dt. Eisenhüttenleute, Düsseldorf

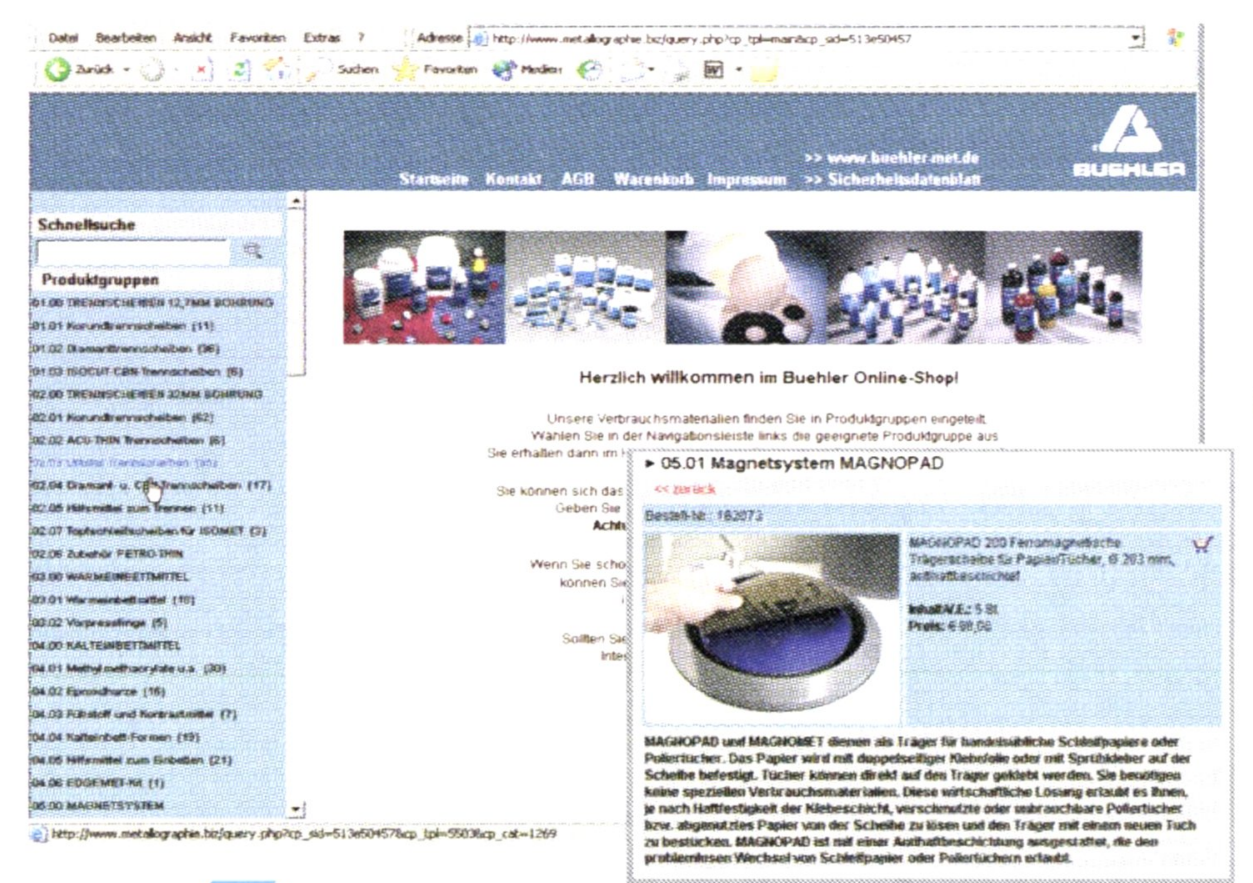

Titelseite: Angelassener Martensit in einem gesinterten Getriebeteil Farbätzung nach Klemm

Polarisiertes Licht, 200x (C) Foto: G. F. Vander Voort Buehler Ltd., USA

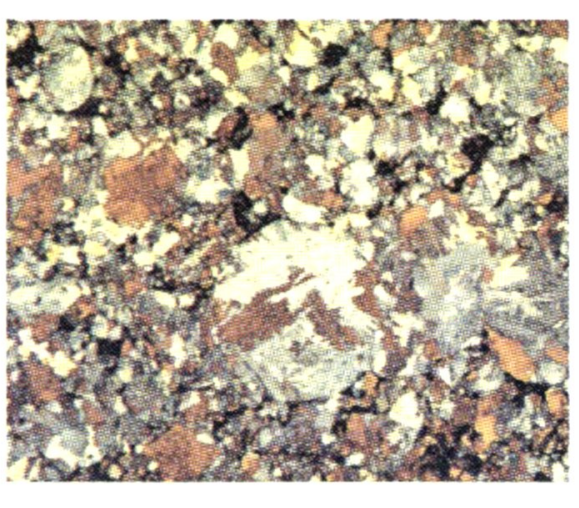

In unserem neu eingerichteten Webshop sind wir rund um die Uhr für Sie da. Besuchen Sie uns: 